\title{
Sintering of ultra high molecular weight polyethylene
}

\author{
SANGEETA HAMBIR and J P JOG* \\ Chemical Engineering Division, National Chemical Laboratory, Pune 411 008, India
}

MS received 13 December 1999

\begin{abstract}
Ultra high molecular weight polyethylene (UHMWPE) is a high performance polymer having low coefficient of friction, good abrasion resistance, good chemical resistance etc. It is used in shipbuilding, textile industries and also in biomedical applications. UHMWPE is processed by powder processing technique because of its high melt viscosity at the processing temperature. Powder processing technique involves compaction of polymeric powder under pressure and sintering of the preforms at temperature above its melting point. In this study, we report our results on compaction and sintering behaviour of two grades of UHMWPE with reference to the powder morphology, sintering temperatures and strength development.
\end{abstract}

Keywords. UHMWPE; powder compaction; sintering; coalescence; strength development.

\section{Introduction}

Powder processing technology is a well-established technique in a variety of fields such as metallurgy, ceramics and pharmaceutical. This technique is also used for processing of very high molecular weight polymers such as polytetrafluoroethylene (PTFE) and ultra high molecular weight polyethylene (UHMWPE). Conventional processing methods such as injection molding cannot be used for these polymers because of their high melt viscosities at the processing temperatures. As a result, these polymers are processed by powder processing technique, which involves cold compaction of the polymeric powders followed by sintering of the preforms at elevated temperatures. The properties of such products are governed by a number of process variables employed during the compaction and sintering processes. The compaction involves pressing the polymeric powder in a closed rigid die. During the compaction process, particle rearrangement, elastic deformation at contact points and finally the compression of the material takes place. The important process parameters are the compaction pressure and the rate of application of pressure.

The sintering process consists of heat treatment of the compacted polymer product or the preform as per a welldefined temperature program. It is an important process as the physical phenomena such as melting, coalescence and crystallization take place during sintering. Accordingly, the temperature and time of the programs are defined taking into consideration these various physical processes. In addition to these process parameters, the other factor that affects the performance of the products is powder charac-

\footnotetext{
*Author for correspondence
}

teristics. Powder characteristics are described in terms of size, size distribution, surface of the particles and shape of the particles as well as powder flow, apparent density, tap density, compactibility etc. There have been various attempts aimed at studying the different aspects of powder processing of polymers and have been discussed in detail elsewhere (Jog 1993).

UHMWPE has received considerable attention during past few years because of its unique combination of properties such as high abrasion resistance, low coefficient of friction, good chemical and stress crack resistance and exceptional resistance at cryogenic temperatures. It is mainly used in electrical industries, shipbuilding, coal and mining, textile industries and even for biomedical applications. There have been few reports on the study of processing of UHMWPE with special reference to particle morphology and effect of metal additives (Halldin and Kamal 1977; Truss et al 1980; Krishnamurthy and Kamal 1989). However, investigations on various aspects of powder processing of UHMWPE are seldom reported.

In this communication, we present results of study of the compaction, sintering process and strength development in UHMWPE samples. Two samples from two different sources have been studied. The differences in the results have been explained on the basis of the powder characteristics and sintering behaviour.

\section{Experimental}

\subsection{Materials}

Two grades of UHMWPE used for the present investigation are Pilene Ultra 1900 and Stamylan UH 210, supplied by Polyolefins Industries, Ltd., and DSM Limburg 
bv, respectively. The properties of these polymers as per data sheets are presented in table 1.

\subsection{Thermal analysis}

A Perkin Elmer DSC 2 equipped with Thermal Analysis Data Station (TADS) was used to characterize the powders for the melting and crystallization behaviour. The melting and crystallization parameters were determined by recording DSC scans at heating and cooling rates of $10^{\circ} \mathrm{C} / \mathrm{min}$ in nitrogen atmosphere. The temperature and energy scales were calibrated using standard procedures.

\subsection{Particle morphology}

The particle morphology of the polymers was studied using scanning electron microscope, model Leica Stereoscan 440 .

\subsection{Compaction}

A carver laboratory Hydraulic press model 25-T was used for compaction of the polymeric powders. A weighed amount of powder $(\sim 1 \cdot 0 \mathrm{~g})$ was compacted into disc specimens of $22 \mathrm{~mm}$ diameter using a cylindrical die. The compaction pressures were varied from $49 \mathrm{MPa}$ to $206 \mathrm{MPa}$. During compaction, the pressure was gradually raised to the maximum and held at that pressure for a dwell time of $3 \mathrm{~min}$. The pressure was then released to the ambient in about $30 \mathrm{sec}$.

Since the polymers are visco-elastic, sufficient time was given for the polymer to relax after compaction. Thus, the dimensions of the compacted pellets were measured after $24 \mathrm{~h}$. From the measurement of dimensions, the density of each pellet was calculated.

\subsection{Sintering}

The sintering behaviour of UHMWPE powder was studied using Leitz polarized light microscope equipped with hot stage. The process of coalescence was experimentally followed by monitoring the neck formation between two polymer particles in contact with each other

Table 1. Properties of UHMWPE samples*.

\begin{tabular}{llll}
\hline Property & Unit & Pilene Ultra & Stamylan \\
\hline Density & $\mathrm{g} / \mathrm{cc}$ & 0.935 & 0.928 \\
Molecular weight & & $3-6 \times 10^{6}$ & $2-6 \times 10^{6}$ \\
Tensile strength & $\mathrm{MPa}$ & $<40$ & 40 \\
Elongation & $\%$ & $<350$ & 450 \\
Notched impact strength & $\mathrm{kJ} / \mathrm{m}^{2}$ & $\mathrm{NF}$ & 160 \\
Shore hardness & & 65 & 62 \\
\hline
\end{tabular}

*As per company data sheet; NF, no failure. as shown in figure 1 . The radia of the polymer particle and the neck were measured using the microvid software provided with the microscope. The diameters of the polymer particles were in the range of $50-100 \mu \mathrm{m}$. The measurements were carried out at five different isothermal temperatures in the range of $170-220^{\circ} \mathrm{C}$.

\subsection{Strength development}

Dumbbell shaped tensile specimens were compacted at $98 \mathrm{MPa}$ pressure and sintered in the mold at $180^{\circ} \mathrm{C}$ for $20 \mathrm{~min}$. The mold was introduced in a preheated press and after completing the dwell time, the mold was cooled by water circulation. These samples were then tested on Instron tensile tester, model 4204 at a crosshead speed of $100 \mathrm{~mm} / \mathrm{min}$.

\section{Results and discussion}

\subsection{Compaction}

As the polymer powder is compacted under pressure, the particles deformed leading to decrease in porosity and increase in density. The process of void reduction has been divided into two independent processes. The first is the filling of holes of the same order of size as the original particles. This occurs by particles sliding past one another accompanied by elastic deformation or slight fracture. The second process concerns the filling of voids that are smaller than the original particles. These can be filled only by plastic flow or by fragmentation. However, as the pressure is released, the elastic component of the strain recovers leading to an increase in volume and thus lower density. This density of the compacts is known as the green density, which is always lower than the theoretical one. Figure 2 illustrates variation of green density with compaction pressure for the two samples. In case of Pilene ultra, it is observed that the green density of the compacts varies with compaction pressure at first rapidly

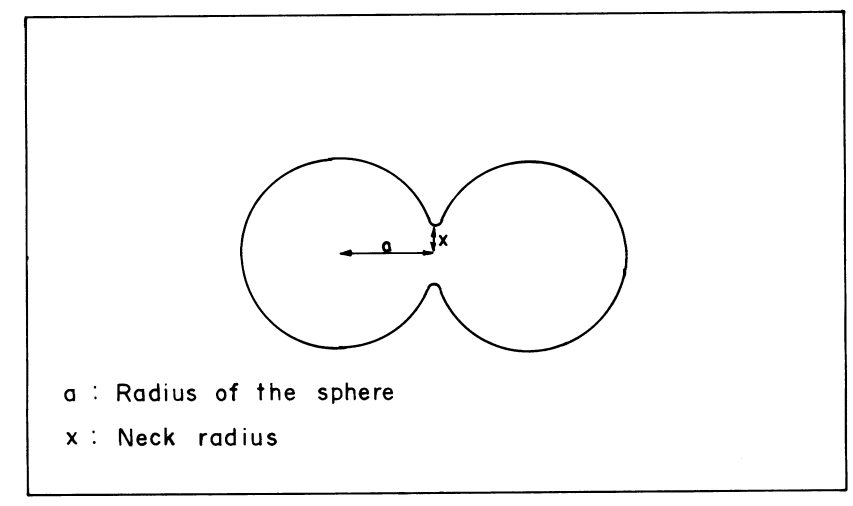

Figure 1. Schematic representation of coalescence of two particles. 
followed by slow increase until it reaches a plateau value. On the other hand, for Stamylan sample the variation of green density with compaction pressure was found to be less significant. As a result the maximum green density, which could be achieved, was about $0.83 \mathrm{~g} / \mathrm{cc}$ and 0. $64 \mathrm{~g} / \mathrm{cc}$ for Pilene ultra and Stamylan samples, respectively.

The particle morphology of the two UHMWPE samples was studied using scanning electron microscope (SEM). Figure 3 illustrates the scanning electron micrographs for the two powders at two magnifications. It is observed that Pilene ultra particles are spherical in shape and exhibit rough surface consisting of nodules connected through fibres. On the other hand, Stamylan particles are spherical in shape with very smooth surface. Compaction behaviour is affected by compaction parameters and particle characteristics (Crawford 1986). Crawford showed that the irregular shaped PP particles could compact very well than that of spherical PP particle. In the present study, since both samples are compacted under identical conditions, the observed differences in their green densities may be ascribed to the differences in the particle characteristics.

\subsection{Sintering}

Sintering involves heat treatment of the compacts as per a well-defined temperature program. It is an important process in processing of polymeric powders as various physical processes take place during this stage. The physical processes, which take place during sintering include melting of the polymer, coalescence of the particles and

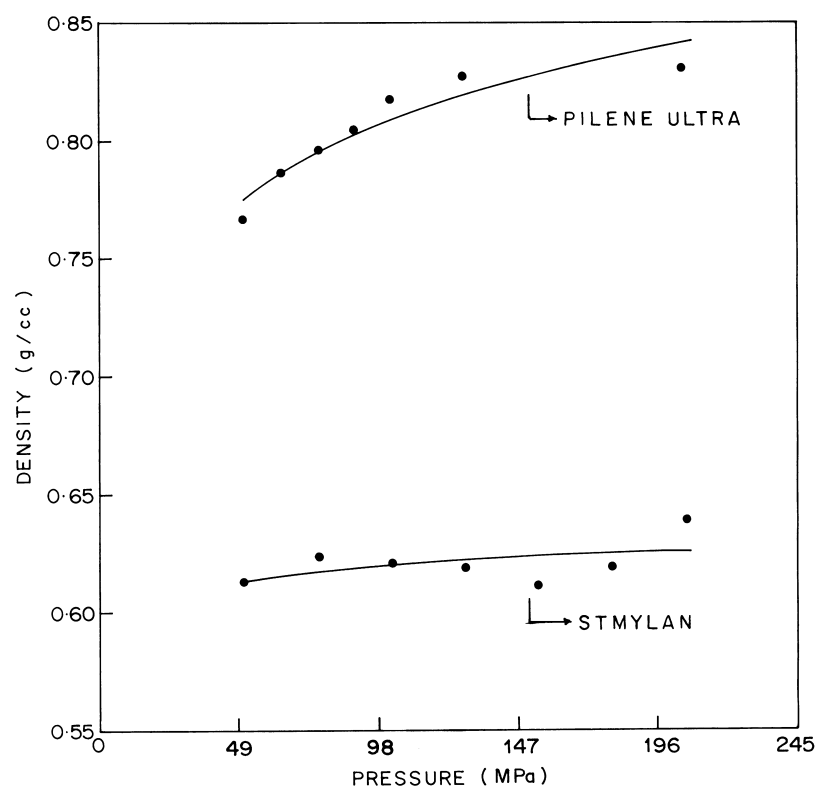

Figure 2. Variation of green density with compaction pressure for UHMWPE powders. and crystallization of the polymer. The information regarding melting and crystallization processes can be generated under isothermal and non-isothermal conditions using thermal analysis techniques. The process of coalescence can be studied at isothermal temperatures using an optical microscopy technique. The information generated during such experiments is useful for optimizing the sintering cycle.

3.2a Melting and crystallization: The melting and crystallization parameters for the two samples are presented in table 2, showing that values of melting point and heat of fusion of Pilene ultra are higher than Stamylan. These higher values indicate the presence of more perfect crystals and a higher degree of crystallization. Since the two polymers are used as received, the changes in the values of melting point and heat of fusion suggest differences in the crystallization of the two samples during polymerization. The crystallization and second heating parameters are however comparable for both the samples, indicating that the crystallization from melt is comparable. Thus the two samples would not exhibit significant differences in the melting and crystallization process during sintering under a given set of conditions.
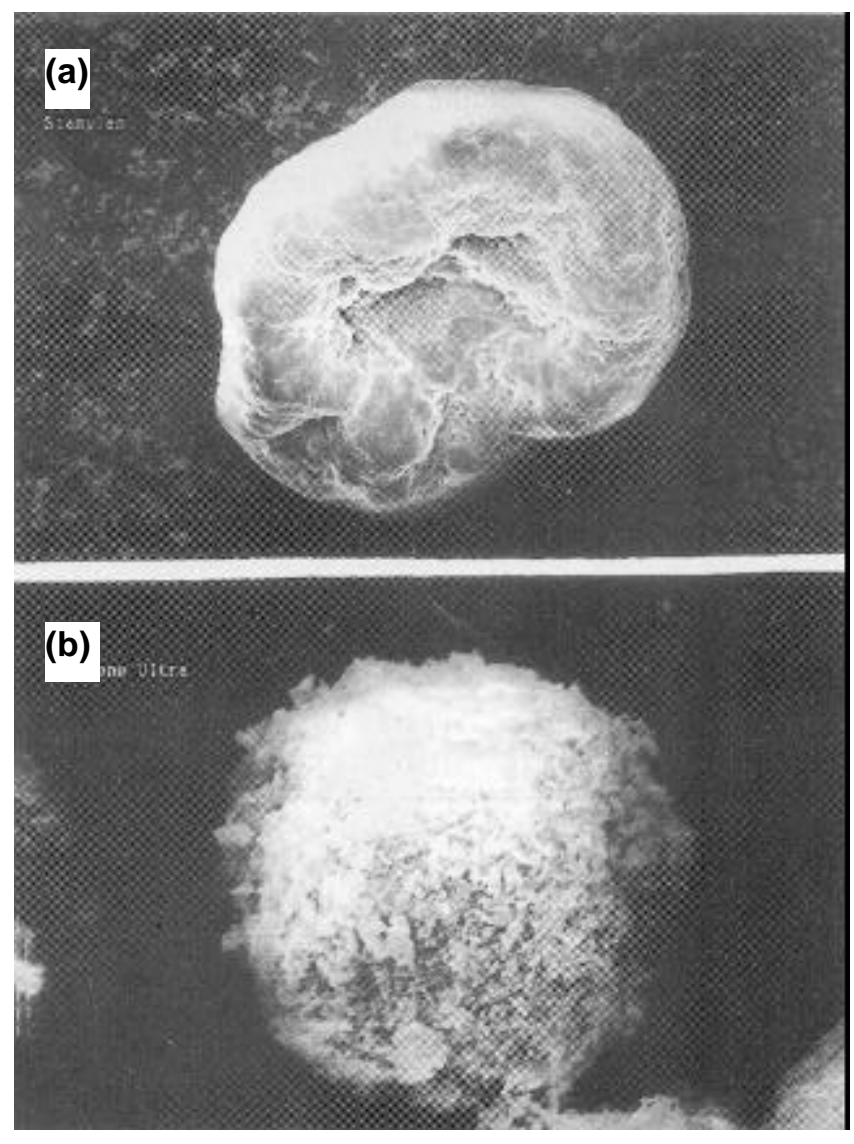

Figure 3. Scanning electron micrographs of two powders a. Stamylan $(\times 500)$ and b. Pilene Ultra $(3 \cdot 5 \mathrm{KX})$. 
3.2b Coalescence: The coalescence of UHMWPE particles was studied following the increase in the neck radius between the particles as a function of time at a constant sintering temperature (figure 1). The sintering behaviour of the two powders can be compared by calculating the time required to reach a certain value of $(x / a)$. Table 3 represents the time required at three sintering temperatures for the two samples to reach the value of $x / a$ to 0.5 . It is observed that for a constant value of $x / a$, the time required for Stamylan is less than that of Pilene ultra indicating that the sintering rate of Stamylan is faster than that of Pilene ultra. However, at high temperature $\left(216^{\circ} \mathrm{C}\right)$, the rates are comparable.

The coalescence of spherical particles has been often explained on the basis of Frenkel's (1945) equation.

A simple theoretical model was developed by Frenkel to describe the early stages of viscous coalescence. He explained the welding of two spheres by Newtonian viscous flow by equating the change in the surface free energy with the viscous dissipation resulting in the following equation:

$$
x^{2} / a=3 / 2 * \gamma * t / \eta
$$

where, $x$ is the neck radius, $a$ the initial radius of the sphere, $\gamma$ the surface tension and $\eta$ the viscosity of the medium. This model assumes that the coalescence occurs by mutual inter-penetration of chains and the radius increases since the volume of the two incompressible particles is constant.

Analysis of the experimental data using Frenkel's equation is illustrated in figure 4 . The variation of $x^{2} / a$ with sintering time at the sintering temperature for Pilene ultra and Stamylan samples show straight lines having the slopes in the range of 1. 8-4. 5. As per Frenkel's equation,

Table 2. Thermal parameters of UHMWPE samples.

\begin{tabular}{|c|c|c|c|c|c|c|}
\hline \multirow[b]{2}{*}{ Sample } & \multicolumn{2}{|c|}{ First heating } & \multicolumn{2}{|c|}{ Cooling } & \multicolumn{2}{|c|}{ Reheating } \\
\hline & $T_{\mathrm{m}}\left({ }^{\circ} \mathrm{C}\right)$ & $\begin{array}{c}\Delta H_{\mathrm{f}} \\
(\mathrm{cal} / \mathrm{g})\end{array}$ & $T_{\mathrm{c}}\left({ }^{\circ} \mathrm{C}\right)$ & $\begin{array}{c}\Delta H_{\mathrm{c}} \\
(\mathrm{cal} / \mathrm{g})\end{array}$ & $T_{\mathrm{m}}\left({ }^{\circ} \mathrm{C}\right)$ & $\begin{array}{c}\Delta H_{\mathrm{f}} \\
(\mathrm{cal} / \mathrm{g})\end{array}$ \\
\hline \multicolumn{7}{|l|}{ Pilene } \\
\hline Ultra & 146 & $48 \cdot 3$ & 112 & $38 \cdot 4$ & 130 & 39. 7 \\
\hline Stamylan & 143 & $45 \cdot 0$ & 109 & $37 \cdot 2$ & 129 & $38 \cdot 7$ \\
\hline
\end{tabular}

Table 3. Sintering time at $x / a=0 \cdot 5$.

\begin{tabular}{lcc}
\hline & \multicolumn{2}{c}{ Sintering time $(\mathrm{sec})$} \\
\cline { 2 - 3 } Temperature $\left({ }^{\circ} \mathrm{C}\right)$ & Stamylan & Pilene Ultra \\
\hline 180 & 224 & 328 \\
187 & 99 & 196 \\
207 & 45 & 60 \\
216 & 20 & 20 \\
\hline
\end{tabular}

the slope should be about one. The higher values of slopes indicate the deviation from Frenkel's equation. Similar observations are reported by Kuczynski et al (1970) in case of PMMA, which were ascribed to the change in flow behaviour of PMMA. The deviations could also be due to the simplicity of the model, which does not take into account the effect of the particle morphology as reported by Siegmann et al (1986) in case of polyethylenes. Thus it seems that the experimental data in the present investigation cannot be represented by a simple equation as suggested by Frenkel.

It is well known that, when two similar polymer surfaces are brought into contact with each other above the
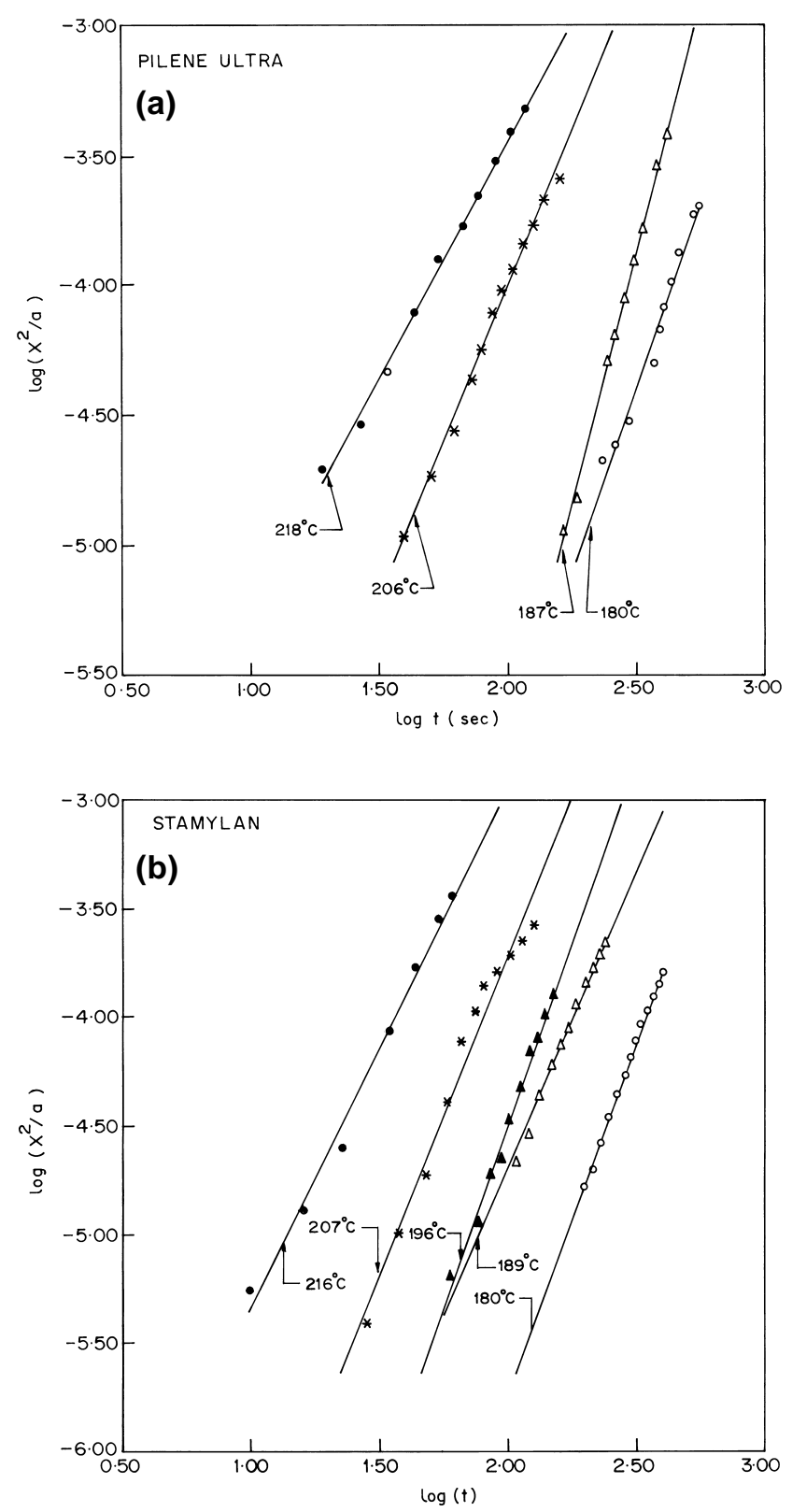

Figure 4. Variation of $x^{2} / a$ with sintering time a. Pilene Ultra and b. Stamylan. 
glass transition temperature, the interface gradually disappears and mechanical strength at the interface develops due to diffusion of polymer chains (Kim and Wool 1983). It can be visualized that a similar process takes place during sintering. The process of coalescence is similar to the process of healing. Thus the process can be defined on the basis of healing theories put forward by Wool et al (1989). According to Wool, the macroscopic behaviour of the interface in terms of mechanical properties is determined by the average interpenetrating contour length $l(t)$, which increases with square root of time. In coalescence also the mechanical strength is a result of diffusion of
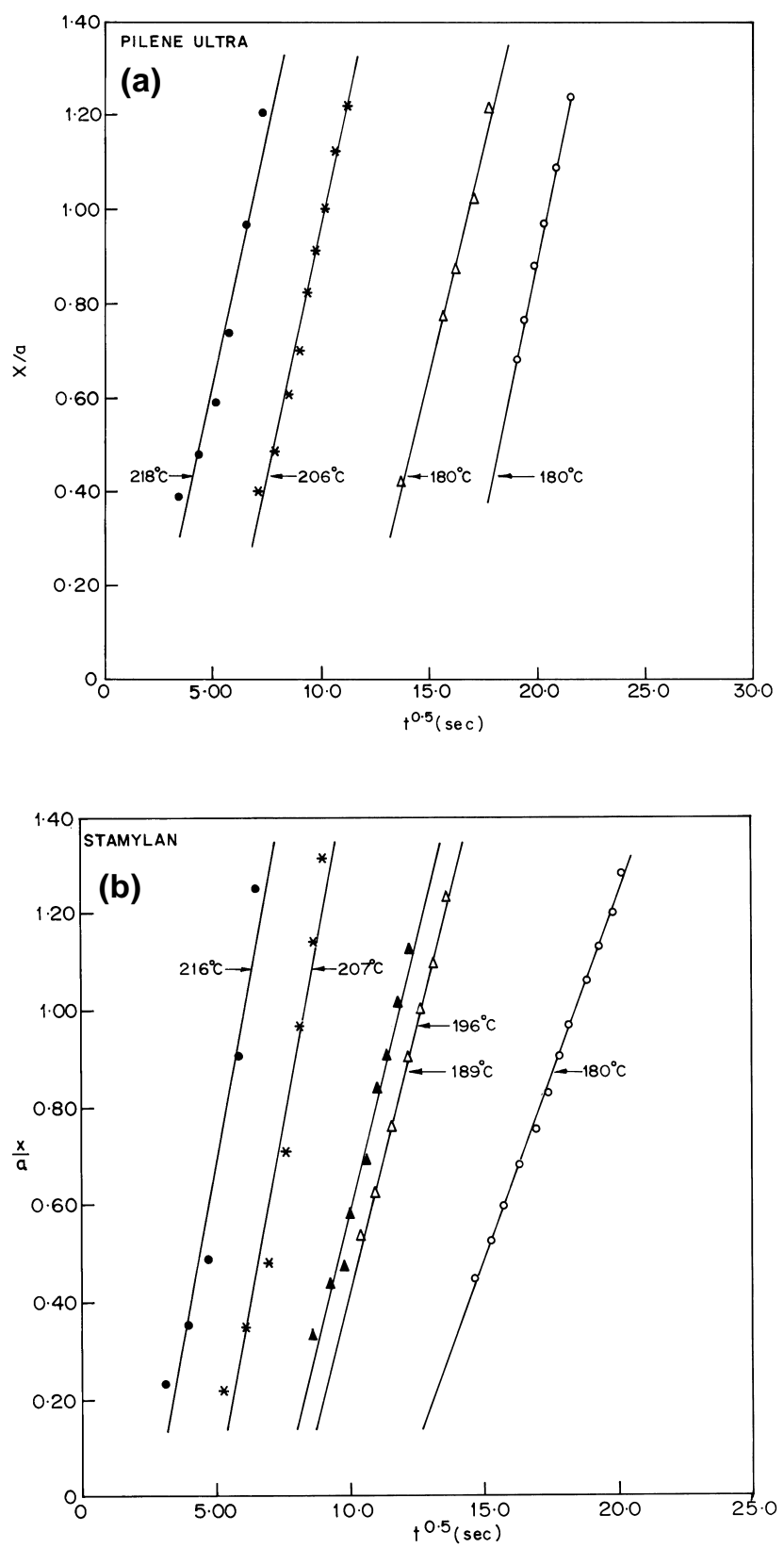

Figure 5. Variation of $x / a$ with $t^{0.5}$ a. Pilene Ultra and b. Stamylan. polymer chains across the interface. Thus the time dependence of $x / a$ can be explained on the basis of interface development and its disappearance between the polymer particles. Figure 5 illustrates the dependence of $x / a$ on $t^{0.5}$. The linearity of the curves confirms the application of the diffusion theory to coalescence process.

3.2c Morphology: The optical micrographs of the particles of Stamylan after coalescence are shown in figure 6. It is seen from the photographs that during the sintering of particles, the rearrangement of the crystallites takes place only near the neck and at the periphery. The nature of the microstructure at the interface reveals that the crystallization of the polymer takes place from an isotropic melt except at the interface. A distinct trans-crystalline region is readily observed at the interface indicating preferred orientation of the chains.

3.2d Strength development: As discussed earlier, the two polymers exhibit differences in the green density and sintering rate. Since the mechanical properties of the
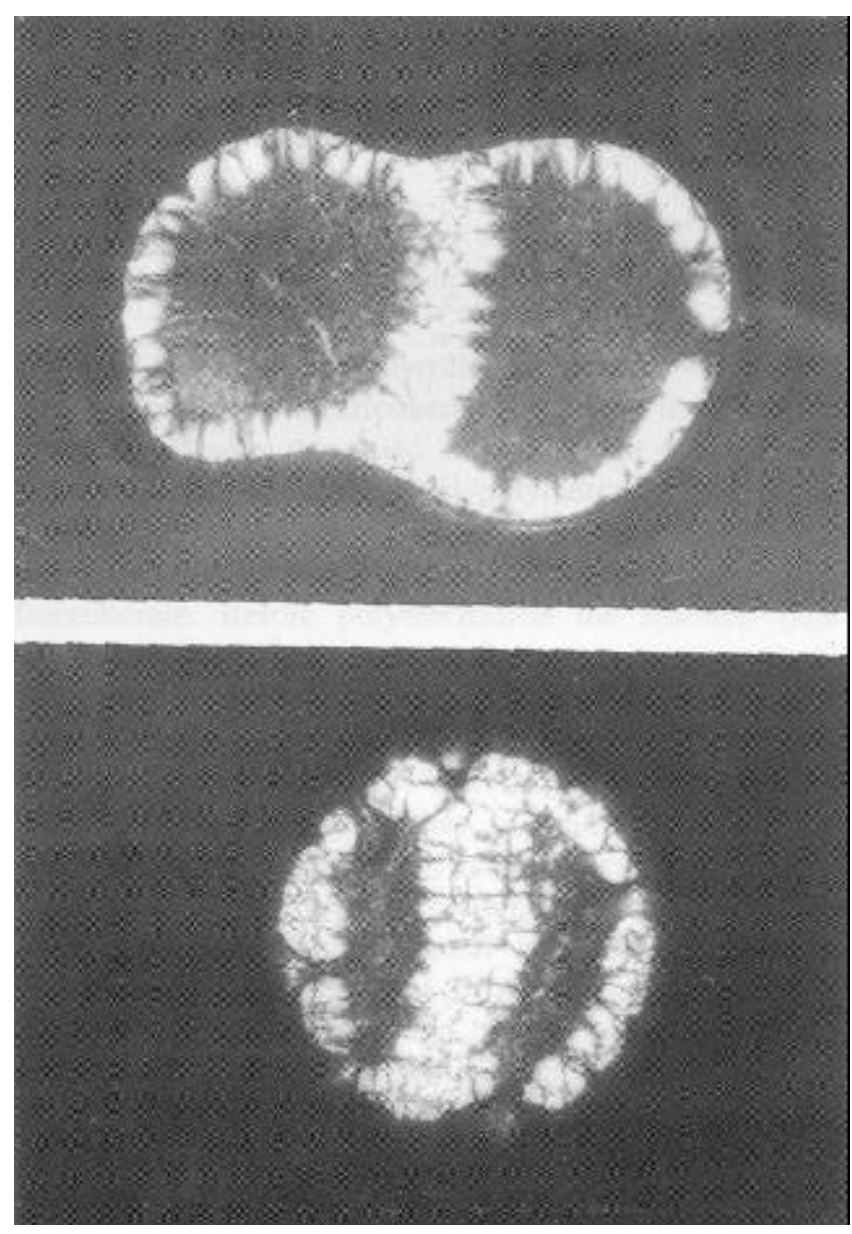

Figure 6. Optical micrographs of polymer powders after coalescence. 
sintered products are governed by the compaction and sintering processes, the differences in the compaction and sintering behaviour would be reflected in the mechanical properties of the samples. Accordingly, experiments were conducted to measure the mechanical strength developed in sintered UHMWPE samples.

The values of tensile strength were found to be 16. $5 \mathrm{MPa}$ for Stamylan and 23. $8 \mathrm{MPa}$ for Pilene Ultra. The density of the compacts of Pilene ultra was higher than that of Stamylan. The higher density of the compacts of Pilene ultra suggests a lower void content and thus the more area of contact between the particles. Thus the mechanical strength would be more for Pilene Ultra than for Stamylan.

The results of the present investigation indicate that the density of the compacted specimens is an important parameter as it determines the contact area between the particles. Thus, the lower value of tensile strength for Stamylan can be ascribed to its lower green density as compared to Pilene Ultra.

\section{Conclusions}

The results of the present investigation can be summarized as follows. Two UHMWPE grades exhibit differences in green densities, sintering behaviour and ultimately in the strength development process. These differences arise due to difference in the particle characteristics, which lead to lower green density. The coalescence process is studied and has been explained on the basis of healing in polymer interfaces. The strength development resulting after sintering is found to depend upon the particle characteristics and green density of the compacts.

\section{References}

Crawford R W 1986 Developments in plastic technology-3 (eds) A Whelan and J L Craft (New York: Applied Science Pub.)

Frenkel J 1945 J. Phys. USSR 9385

Halldin G W and Kamal I L 1977 Polym. Eng. Sci. 1721

Jog J P 1993 Adv. Polym. Tech. 12281

Kim Y H and Wool R P 1983 Macromolecules 131115

Krishnamurthy V and Kamal I L 1989 Polym. Eng. Sci. 29564

Kuczunski G C, Neuville B and Toner H P 1970 J. Appl. Polym. Sci. 142069

Siegmann A, Raiter I and Narkis M 1986 J. Mater. Sci. 211180 Truss R W, Han K S, Wallace J F and Geil P H 1980 Polym. Eng. Sci. 20747

Wool R P, Yuan B L and McGarel O J 1989 Polym. Eng. Sci. 291340 